Check for updates

Cite this: RSC Adv., 2017, 7, 56648

Received 29th October 2017

Accepted 11th December 2017

DOI: 10.1039/c7ra11910k

rsc.li/rsc-advances

\section{Fabricating rutile nanopins on an anatase hollow sphere structure with enhanced photoactivity performance $\dagger$}

\begin{abstract}
Sheng-Cai Zhu (iD ${ }^{b}$ and Ling Fu*a
$\mathrm{TiO}_{2}$ is a promising material for the renewable energy and pollution control field. Hierarchically, structure and phase junction are vitally important to circumvent the limited photo adsorption and charge separation. In this work, we successfully synthesized a hierarchical structure $\mathrm{TiO}_{2}$ with tunable rutile nanopins on anatase hollow spheres using a facile one-step synthesis method. The phase composition and structure can be controlled by sulfate, chloride ions and sodium dodecyl sulfate (SDS). Systematic density functional theory (DFT) was used to study the interface of an anatase/rutile structure and energetic origins of the effects of the sulfate and chloride ions on anatase (101) and rutile (110) surfaces. The atomic junction structure in this work may help to obtain a comprehensive understanding of the interface. The higher photoactivity performance can be attributed to the multi-reflections of electromagnetic waves and efficient charge separation. The hierarchical structure of rutile nanopins at anatase hollow microspheres may have great potential for practical applications.
\end{abstract}

\section{Introduction}

Semiconductors are promising materials for storing solar energy and removing pollutants from the environment. Among various semiconductors, titanium is the most widely investigated in many photocatalytic processes, such as water splitting, ${ }^{1-3} \mathrm{CO}_{2}$ reduction ${ }^{4,5}$ etc., due to its high stability, nontoxicity and low cost. ${ }^{6}$ However, the application of $\mathrm{TiO}_{2}$ as a photocatalyst is limited by its low activity. Further improving the photoactivity of $\mathrm{TiO}_{2}$ is essential for its practical application.

Abundant evidence has shown that $\mathrm{TiO}_{2}$ particles with specific morphology would have enhanced photoactivity compared to their random counterparts. ${ }^{7-12}$ Controlling the morphology is a crucial issue in the synthesis of materials. In the last decade, various hierarchical architectures, e.g. nanoflowers, nanospheres and nanoboxes, have been successfully achieved in oxides, ${ }^{13-20}$ and researchers have shown that these

\footnotetext{
${ }^{a}$ Henan Key Laboratory of Ecological Security for Water Source Region of Mid-line of South-to-North Diversion Project of Henan Province, Collaborative Innovation Center of Water Security for Water Source Region of Mid-line of South-to-North Diversion Project of Henan Province, College of Agricultural Engineering, Nanyang Normal University, Nanyang 473061, P. R. China. E-mail: ful@nynu.edu.cn

${ }^{b}$ Center for High Pressure Science and Technology Advanced Research (HPSTAR), Shanghai 201203, P. R. China

$\dagger$ Electronic supplementary information (ESI) available: The calculation detail; the superlattice biphase crystal and the energy of anatase/II and II/rutile ratio; the atomic model of HRTEM and the dihedral angles of anatase planes and rutile planes; the TEM images of the hierarchical at different time and the SEM of $\mathrm{TiO}_{2}$ synthesis at other parameters; the average adsorption energy of $\mathrm{Cl}^{-}$on anatase (101) and rutile (110) surface. See DOI: 10.1039/c7ra11910k
}

architectures can promote the photoactivity. ${ }^{21-23}$ For example, $\mathrm{Li}^{24}$ reported that the hierarchical structure shows a much better photocatalytic performance than its irregular dimensional counterparts in phenol degradation. The hierarchical architectures effectively enhance light scattering and trapping for efficient photon harvesting and thus promote the photoadsorption. $^{25}$

Phase junction is another approach to promote the photoactivity. ${ }^{26,27}$ Most $\mathrm{TiO}_{2}$-based single phase photocatalysts show low efficiency, due to its limited charge separation. Recent studies have shown that phase junction could provide efficient charge separation. ${ }^{28,29}$ The $\mathrm{TiO}_{2}$ phase junction photocatalyst composites with multiple phases, such as anatase/rutile, ${ }^{30,31}$ $\mathrm{TiO}_{2}-\mathrm{B} /$ anatase $^{32-35}$ and rutile/brookite, ${ }^{36}$ show synergistically work which yield higher photoactivity for a wide range of reactions. For instance, Kawahara ${ }^{28}$ found the bilayer anatase/rutile film $\mathrm{TiO}_{2}$ photocatalyst possesses higher photocatalytic activity for $\mathrm{CH}_{3} \mathrm{CHO}$ oxidation than the individual one. Also, our recent calculation work has shown that hole and electron can be efficient separated at the anatase/rutile interface. ${ }^{37}$ The discovery of higher performance of phase junction than single phase has greatly enlightened the effort to design bi-phase materials.

To date, hierarchical structure usually contains only one single phase, and few hierarchical architectures of bi-crystalline materials have ever been successfully synthesized. ${ }^{38-41}$ The aim of this work is to synthesize a bi-crystalline $\mathrm{TiO}_{2}$ structure which combines the advantages of hierarchical structure and phase junction. By hydrothermal method with sodium dodecyl sulfate (SDS) colloidal microspheres as soft template, dendritic rutile nanopins on anatase hollow spheres was synthesized, which the 
rutile in the nanorod anchored on the hollow spheres surface. The key point to fabricate of the phase hierarchical structure is to control the surfactant self-aggregation structure and concentrations of adsorption agents. By using the angledependent HRTEM characterization and combine with the DFT result, the junction structures were studied. The formation mechanism was studied by the time dependent morphology evolution. The hierarchical bi-crystalline fabricated in this study are of great interest not only for its morphology but also the remarkable photoactivity.

\section{Experimental and theoretical methods}

\section{Preparation of the hierarchical bi-phase $\mathrm{TiO}_{2}$}

The bi-crystalline $\mathrm{TiO}_{2}$ hierarchical structure is synthesized using a one-pot hydrothermal method as described elsewhere with some modification. ${ }^{42}$ Typically, 0.012 M SDS (99.9\%; supplied by Alfa Aesar) was slowly added into the deionized water with strong stir. Then, $0.15 \mathrm{M} \mathrm{TiCl}_{3}$ solution was prepared by dissolving $\mathrm{TiCl}_{3}(20 \mathrm{w} \%$, in $3 \% \mathrm{HCl}$ dilute aqueous; supplied by Alfa Aesar) in above SDS solution during stirring. After stir for $100 \mathrm{~min}$, the achieved violet microemulsion $(0.07 \mathrm{~L})$ was poured into a Teflon-lined stainless steel autoclave $(100 \mathrm{~mL})$ and placed into an oven maintaining $200{ }^{\circ} \mathrm{C}$ for $3 \mathrm{~h}$. The oven was then cooled to room temperature in air. The collected precipitates were centrifuged and washed repeatedly by deionized water for 3 times and by ethanol for 2 times to remove the surfactant and/ or inorganic reagents. Finally, the samples were dried at $80{ }^{\circ} \mathrm{C}$ overnight.

\section{Characterization}

The morphology of products was characterized by scanning electron microscopy (FESEM, FEI Nova NanoSEM450, USA, operated at $2-5 \mathrm{kV}$ ). The microstructure of the products was investigated by TEM and HRTEM (FEI Tecnai G2F20 S-Twin, USA) operated at $200 \mathrm{kV}$. The selected area electron diffraction patterns (SAED) of the samples were recorded on an electron microscope operated at $200 \mathrm{kV}$. The analysis of the crystal phases of all samples were conducted on a Bruker D8 Advance diffractometer using $\mathrm{Cu} \mathrm{K} \alpha$ radiation at a scanning rate of $8^{\circ} \mathrm{min}^{-1}$ in the range of $20-90^{\circ}$ at room temperature.

\section{Photocatalytic activity measurements}

The activity of hierarchical bi-phase $\mathrm{TiO}_{2}$ material was probed by photodegradation of methylene blue (MB) dyes. In a typical process, aqueous solution of the MB dyes $\left(0.01 \mathrm{~g} \mathrm{~L}^{-1}, \approx 2.7 \times\right.$ $\left.10^{-5} \mathrm{M}, 0.1 \mathrm{~L}\right)$ and the photocatalysts $(0.050 \mathrm{~g})$ were placed in a $0.2 \mathrm{~L}$ glass vessel (the cross sectional area is $50 \mathrm{~cm}^{2}$ ). Before irradiation, the suspensions were stirred in the dark for $4 \mathrm{~h}$ to ensure the establishment of adsorption/desorption equilibrium. Under the ambient conditions and stirring, the photoreaction vessel was exposed to the UV irradiation produced by a $50 \mathrm{~W}$ high pressure Xe lamp with the main wave crest at $365 \mathrm{~nm}$, which was positioned $30 \mathrm{~cm}$ above the vessel (the intensity at the wavelength of $365 \mathrm{~nm}$ at the catalyst mixture surface was $30 \mu \mathrm{W} \mathrm{cm}{ }^{-2}$, estimated with a radiometer). The photocatalytic reaction was started by turning on the Xe lamp, and during the photocatalysis test, all other lights were blocked. At given time intervals, $5 \mathrm{~mL}$ of the sample solution was taken out and centrifuged to remove the catalyst completely. Then the solution was analyzed on a Varian UV-vis spectrophotometer by recording variations of the absorption band of $\mathrm{MB}$ at $660 \mathrm{~nm}$.

\section{Theoretical calculations}

All DFT calculations were performed using SIESTA $^{43}$ where optimized double- $\zeta$ plus polarization numerical atomic orbital basis sets ${ }^{44}$ were utilized along with the Troullier-Martins norm-conserving pseudopotentials. ${ }^{45}$ The exchange correlation functional utilized is at the generalized gradient approximation level proposed by Perdew, Burke and Ernzerhof (GGA-PBE). ${ }^{46}$ The L-BFGS method was employed for geometry relaxation until the maximal forces on each relaxed atom were less than $0.01 \mathrm{eV}$ $\AA^{-1}$. The rutile (110) and anatase (101) surfaces are both modeled by a rectangular unit cell of $6 \mathrm{TiO}_{2}$ layers slab with the lattice parameters of $(14.834 \times 13.145 \AA, 360$ atoms $)$ and $(10.398 \times 15.264 \AA$, 288 atoms), respectively, and the vacuum spacing is generally larger than $30 \AA$. In all calculations, the central two $\mathrm{TiO}_{2}$ layers are fixed at bulk-truncated positions while the other layers are allowed to relax. The adsorption of $\mathrm{SO}_{4}{ }^{2-}$ and $\mathrm{Cl}^{-}$was modeled on sides of the slab, and during optimization, all the slab and adsorbate atoms were allowed to move. The solid-liquid interface model is using a recently-developed periodic CM-MPB method, ${ }^{47,48}$ which can account for the long-range electrostatic interaction due to solvation between surface and solution. This approach was utilized to model electrochemical reaction on metal surfaces ${ }^{49}$ and a detailed description on the methodology can be found in ref. 50 .

The surface energy of clean anatase $\mathrm{TiO}_{2}$ (101) surface was taken from an early DFT study in ref. 51 . The average adsorption energies of $\mathrm{SO}_{4}{ }^{2-}$ and $\mathrm{Cl}^{-}$on surfaces were calculated by using the following equation,

$$
E_{\text {ad }}=-\left(E_{\text {sur }+ \text { ad }}-E_{\text {sur }}-n \times E_{\text {ad }}\right) / n
$$

where $E_{\text {sur }}$ is the energy of the clean $\mathrm{TiO}_{2}$ surface, $E_{\text {surtad }}$ is the total energy of the system with adsorbed molecules at the surface with solvation effect, $E_{\text {ad }}$ is the solvation energies of a single $\mathrm{SO}_{4}{ }^{2-}$ or $\mathrm{Cl}^{-}$ion, and $n$ is the number of adsorbed molecules on the surface. Solvation effect was considered for all the energies.

\section{Results and discussion}

The hierarchical structure of hollow anatase sphere (core)/rutile nanopins (shell) was synthesized using hydrothermal method. The morphology of the anatase/rutile hierarchical structure was studied by electron microscopy. Fig. 1a shows the SEM image of the as-synthesized anatase/rutile hierarchical architectures, with a magnified SEM images as shown in Fig. 1b. It can be found that the anatase/rutile hierarchical architectures are really uniform, the central zone is hollow anatase sphere, and rutile nanopins radiate from the hollow anatase sphere to form the anatase (core)/rutile (shell) system. Most of the product is in 

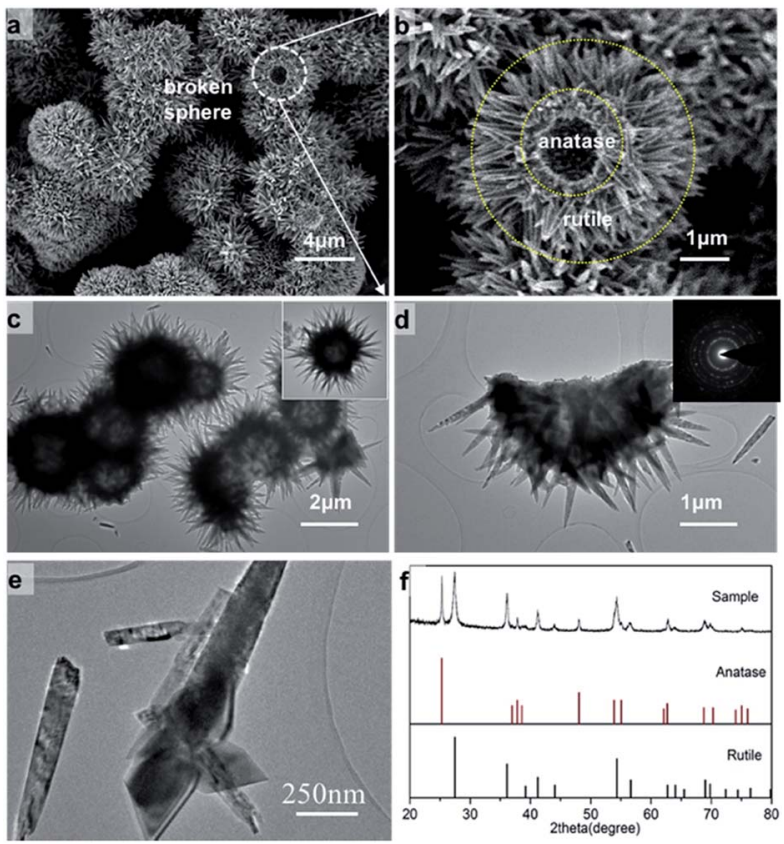

Fig. 1 Hierarchical $\mathrm{TiO}_{2}$ biphase structure imaged with $\operatorname{SEM}(a, b)$ and (c-e) TEM; XRD pattern of hierarchical bi-phase $\mathrm{TiO}_{2}$ structure.

a shape of chestnut, while some spheres are broken (Fig. 1b). For the broken hierarchical architecture as shown in Fig. 1b, the inner diameter of the anatase sphere is $2.5 \mu \mathrm{m}$ with thickness of $0.5 \mu \mathrm{m}$, while $1.2 \mu \mathrm{m}$ long rutile nanopins grow on the outer of the sphere. To provide further insight into the hierarchical biphase structure, analytical TEM investigations were performed. Fig. 1c shows the typical FETEM image, it can be seen that the hierarchical structure are hollow spheres. The single hollow sphere SEAD pattern (inserted in Fig. 1d) shows that the $\mathrm{TiO}_{2}$ contains both anatase and rutile. A TEM image of this biphase junction material is shown in Fig. 1e, where the shape of anatase and rutile structures were nanooctahedral and nanorod, respectively. The rutile nanorods are fixed on the interface of two anatase particles; such T-shaped bi-phase junction has been predicted in our recent work. ${ }^{37}$ However, some of the anatase/rutile junction was broken before dropping the sample to the carbon mesh by ultrasonic preparation. This somehow indicates high strain on the interface of anatase/rutile. Fig. if shows the XRD pattern of the hierarchical architecture. The diffraction peaks of XRD can be well-indexed to anatase and rutile regardless of the intensity, suggesting that the hierarchical structures consist both of anatase and rutile phase.

The hierarchical structure crystallinity is further revealed by angle-dependent HRTEM characterization under various viewing zones, shown in Fig. 2. Meanwhile, theoretical investigation were performed. We built the anatase/II/rutile tri-phase junction particle with orientation relationship $(112)_{\mathrm{A}} / /(100)_{\mathrm{II}}+$ $[-110]_{\mathrm{A}} / /[010]_{\mathrm{II}}$ and $(100)_{\mathrm{II}} / /(200)_{\mathrm{R}}+[001]_{\mathrm{II}} / /[101]_{\mathrm{R}}$ (the subscript $\mathrm{R}$, II, A indicate rutile, $\mathrm{TiO}_{2}$-II and anatase phases, respectively). ${ }^{37,52,53}$ The detail of building the tri-phase particle can be found in Fig. S1 in ESI. $\uparrow$ These tri-phase nanoparticle models were then fully optimized under PBE functional, the geometries

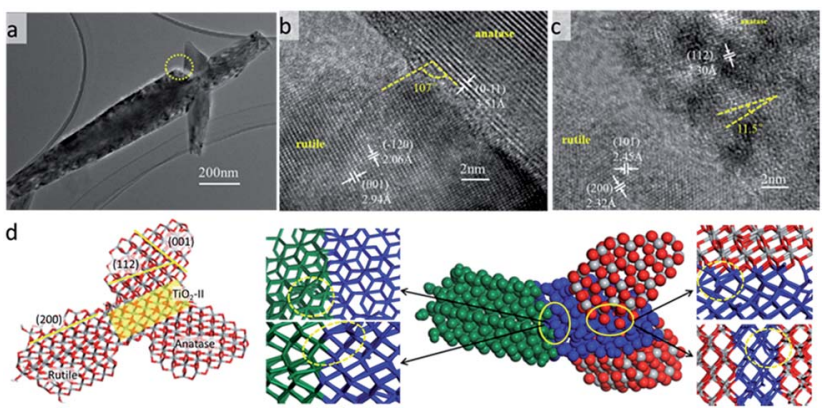

Fig. 2 The TEM and HRTEM characterization of bi-phase junction and theoretical model of tri-phase junction. (a) TEM analysis for anatase/ rutile crystal; ( $b$ and $c$ ) the angle-dependent HRTEM characterization for the circle area in under various viewing angles; the structure of anatase/II/rutile nanoparticle and (d) the interface structure of anatase/ $\mathrm{TiO}_{2}-\|$ and $\mathrm{TiO}_{2}-\| /$ rutile optimized by DFT. Orientation relation is $(112)_{A} / /(100)_{\|,},[-110]_{A} / /[010]_{\|}$and $(100)_{\| \mid} / /(200)_{R},[001]_{\|} / /[101]_{R}$. The yellow dotted circles show the dislocation of the interface zone. Green spheres represent rutile domain, blue spheres represents $\mathrm{TiO}_{2}-\|$ domain, and red and grey spheres represents anatase domain.

are shown in Fig. 2d. As TEM images show, rutile nanopins anchor on the anatase boundary to form a T-shaped bi-phase junction. Different zone-axis patterns of HRTEM originated from the same crystal are illustrated in Fig. $2 b$ and c, and the important data on the inter-planar $d$-spacing and dihedral angles are indicated. Fig. $2 \mathrm{~b}$ shows that the anatase $(0-11)_{\mathrm{A}}$ plane with $d$-spacing $3.51 \AA$, the two of rutile planes are perpendicular to each other with $d$-spacing of $(1-20)_{\mathrm{R}} 2.06 \AA$ and $(001)_{\mathrm{R}} 2.94 \AA$, respectively. These results indicate rutile crystals grew along the [001] direction. Rotating the zone axis, we were able to observe several other surface planes, which can reveal the atomic configurations of the phase junction. The hexagon pattern of rutile can be assigned to (101) and (200) with $d$-spacing $2.45 \AA$ and $2.32 \AA$, respectively. The atomic model compared with every HRTEM image can be found in Fig. S2 in ESI. $\dagger$ The results show that the dihedral angles between $(200)_{R}$ and $(112)_{\mathrm{A}}$ is 11.5 degree, while the theoretically predicted values is zero degree.

This deviation between the TEM images and the atomic model might be attributed to the lattice $10 \%$ mismatch between anatase and $\mathrm{TiO}_{2}$-II. Superlattice bi-phase crystal composing $\mathrm{TiO}_{2}$-II/anatase and $\mathrm{TiO}_{2}-\mathrm{II} /$ rutile enables us to provide insight into the interface energy of the bi-phase crystal. The bi-phase crystals are shown in Fig. S1 in ESI. $\uparrow$ The interface mismatch of II on anatase (112) facet decreases with the decreasing of the number of layers on anatase, but the rutile/II ration has negligible effect on the mismatch. Some dislocation can be found on the interface due to the large strain inside the tri-phase junction particle model shown in Fig. 2d, and the thickness of dislocation region depends on the size of crystals. High strain between anatase and rutile in the experiment is also confirmed by ultra sonication which breaks the anatase/rutile junction.

To provide insight into the mechanism of formation of hierarchical bi-phase, the time-dependent morphology evolution was studied at $200{ }^{\circ} \mathrm{C}$ with $0.1 \mathrm{M} \mathrm{TiCl}_{3}$ and $0.012 \mathrm{M}$ SDS (showed in Fig. 3). When the reaction time only $40 \mathrm{~min}$, as 

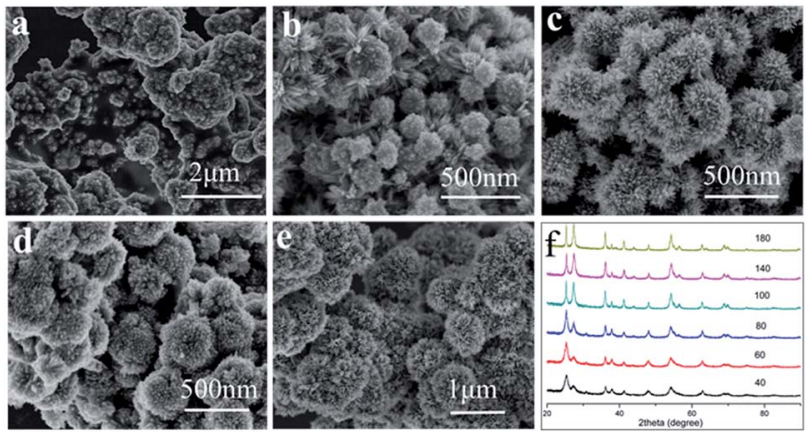

$\mathrm{g}$

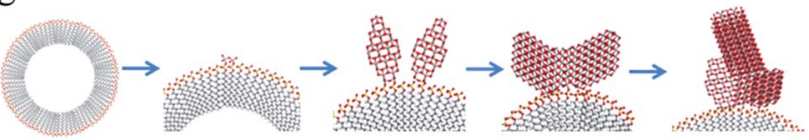

Fig. 3 FESEM images and XRD pattern of the hierarchical anatase (core)/rutile (shell) bi-phase structure grown at $200{ }^{\circ} \mathrm{C}$ with the $\mathrm{TiCl}_{3}$ and SDS concentrations fixed at $0.1 \mathrm{M}$ and $0.012 \mathrm{M}$, respectively, with different synthesis time (a) $40 \mathrm{~min}$, (b) $60 \mathrm{~min}$, (c) $80 \mathrm{~min}$, (d) $100 \mathrm{~min}$, (e) 140 min. (f) XRD pattern of the sample with different time. (g) Schematic illustration of the growth mechanism.

shown in Fig. 3a, the as-synthesized anatase powder is random. By prolong to $60 \mathrm{~min}$, anatase solid hollow spheres were obtained (see Fig. $3 \mathrm{~b}$ and S3 in ESI $\dagger$ ). At the same time, small peaks belong to rutile phase start to appear in XRD pattern which indicates the rutile start to growth when prolong the reaction time. Once formed, rutile nanopins firmly anchor on anatase sphere (as Fig. 3c), and grow quickly with longer reaction time as Fig. 3d and e showed.

Based on the time-dependent morphology evolution, the growth of hierarchical architectures proceeds can be divided into four stages (see Fig. 3g): (1) formation of anatase nucleus on the micelle surface by the titanyl sulfate structure. (2) Then the growth of the initial anatase sphere; in this stage, the anatase octahedral crystals are growth in (001) orientation for the $\mathrm{SO}_{4}{ }^{2-}$; (3) rutile nucleus formation on the grain boundary of anatase; (4) the growth of rutile.

Now, we are at the position to investigate the influence of experimental conditions to the formation of the anatase/rutile bi-phase hierarchical structure. First, we tested the influence of $\mathrm{TiCl}_{3}$ concentration by changing from 0.15 to $0.025 \mathrm{M}$, while SDS concentration was fixed at $0.025 \mathrm{M}$. Fig. 4 depicts influence of $\mathrm{Ti}$ precursor concentration. It can be found that the rutile density and radio reduces when Ti precursor decreasing; and when the $\mathrm{TiCl}_{3}$ concentration is below $0.025 \mathrm{M}$ rutile nanopins disappeared (see Fig. S4 in ESI†). This also can be found in the XRD pattern that only weak rutile (110) peak can be observer, which indicates the proportion of rutile phase decreases while Ti precursor decreases. For the $\mathrm{SO}_{4}{ }^{2-}$ inhibit the growth of rutile, it is reasonable that the Ti precursor decreasing will produce less rutile.

Second, we investigate the mechanism of formation of hollow sphere. $\mathrm{TiCl}_{3}$ concentration was fixed at $0.1 \mathrm{M}$, the decrease of SDS concentration would significantly affect the morphology of hierarchical $\mathrm{TiO}_{2}$. When the SDS concentration was $0.012 \mathrm{M}$, the morphology of bi-phase retains hierarchical
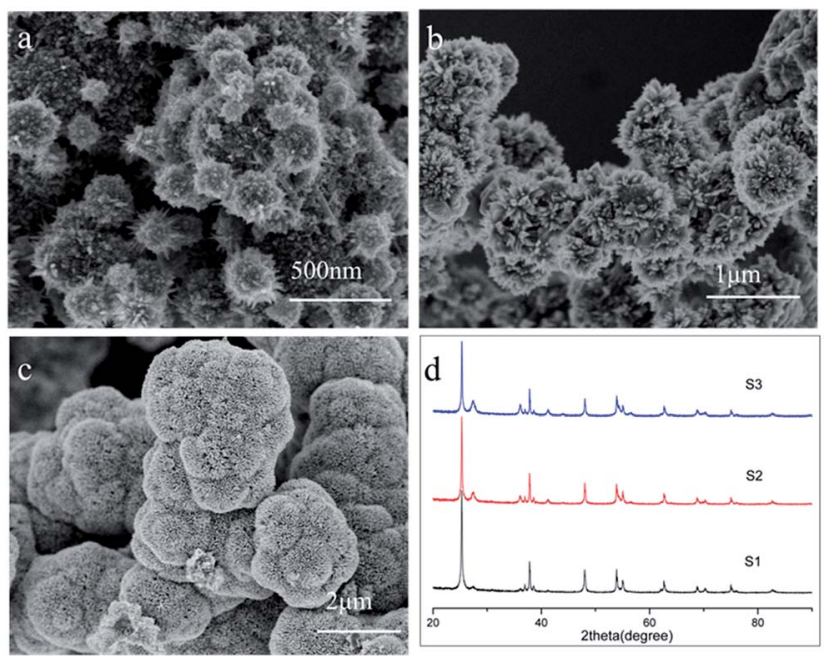

Fig. 4 FESEM images of the bi-phase production grown at $200{ }^{\circ} \mathrm{C}$ for $3 \mathrm{~h}$ with SDS fixed at $0.025 \mathrm{M}$ and $\mathrm{TiCl}_{3}$ concentration varies from (a) $0.05 \mathrm{M}$ (S1), (b) $0.1 \mathrm{M}$ (S2), (c) $0.15 \mathrm{M}$ (S3), and (d) the corresponding $\mathrm{XRD}$ pattern of the bi-phase production.

structure. While SDS concentration changed to $0.006 \mathrm{M}$, the hierarchical structure disappeared, as shown in Fig. 5 and S4.† To our knowledge, surfactant will aggregate to form microsphere in the aqueous solution when the concentration is above critical micelle concentration (CMC). In this work, the CMC of SDS is $0.008 \mathrm{M}$. When the SDS above the CMC, it will aggregate with a configuration of $\mathrm{SO}_{4}{ }^{2-}$ species toward water to form micelle in the solution. ${ }^{54}$ As a result, the negative sulfate group on the microsphere surface attract $\mathrm{Ti}^{3+}$, thus anatase nuclei and grow on the microsphere when the concentration above CMC. ${ }^{55}$ When the concentration is lower than CMC, the SDS is dissolved in water as solution, as a result, anatase and rutile form the irregular powder.
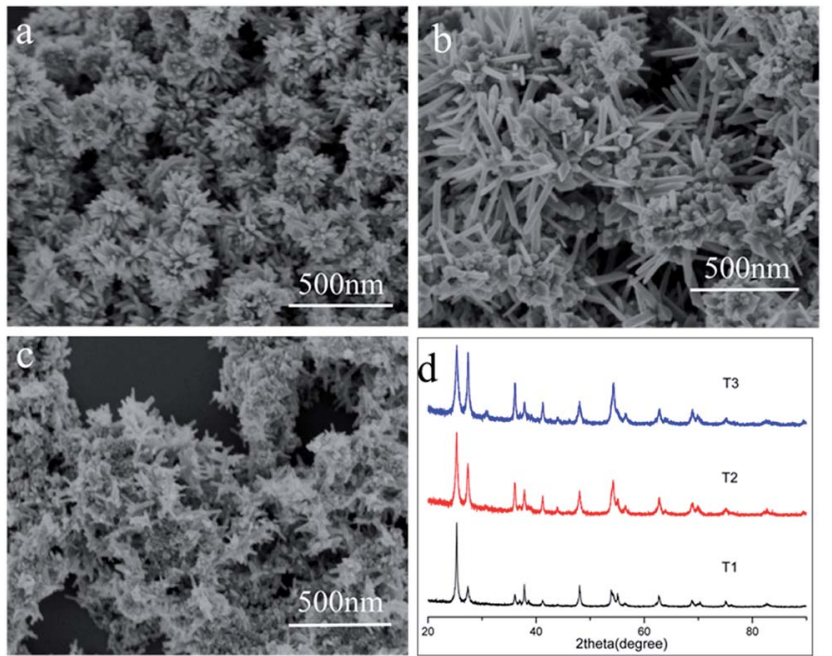

Fig. 5 FESEM images and the corresponding XRD pattern of the biphase production grown at $200^{\circ} \mathrm{C}$ for $3 \mathrm{~h}$ with $\mathrm{TiCl}_{3}$ concentration fixed at $0.1 \mathrm{M}$ and SDS varies from (a) $0.012 \mathrm{M}$ (T1), (b) $0.006 \mathrm{M}$ (T2), (c) $0.003 \mathrm{M}$ (T3). (d) Corresponding XRD pattern of the bi-phase production. 
To understand the atomic mechanisms of experimental results, we performed density functional theory (DFT) calculations to illuminate the energetic profiles of the adsorption energy for $\mathrm{SO}_{4}{ }^{2-}$ and $\mathrm{Cl}^{-}$, the details of calculation can be found in $\mathrm{SI} \mathrm{TiO}_{2}$ surfaces were modeled by periodic slabs, and anatase (101) and rutile (110) surface were used for the calculation because these two facets are the most exposed surface in our experiment. The solvation effect was taken into consideration via the periodic continuum solvation model based on the modified Poisson-Boltzmann equation. ${ }^{48,51}$ We have considered various adsorption configurations for $\mathrm{SO}_{4}{ }^{2-}$ and $\mathrm{Cl}^{-}$, on both surfaces, as shown in Fig. 6. The adsorption energy of $\mathrm{SO}_{4}{ }^{2-}$ on rutile (110) surface $(2.00 \mathrm{eV})$ is much higher than on anatase (101) $(1.41 \mathrm{eV})$. This could be understood that the adsorption configurations of $\mathrm{SO}_{4}{ }^{2-}$ on rutile (110) surface can form two stronger $\mathrm{Ti}-\mathrm{O}$ bonds $(1.989 \AA 2.003 \AA)$ than that on anatase (101) surface $(2.076 \AA, 2.099 \AA)$. The stronger bonding of sulfate species on the rutile (101) surface suppresses rutile growth, which might explain our experimental results. On the other hand, different $\mathrm{Cl}^{-}$ion concentration has dramatic different adsorption energy. When at low $\mathrm{Cl}^{-}$ion concentration, e.g., one $\mathrm{Cl}^{-}$ion $(0.70 \mathrm{eV})$ and two $\mathrm{Cl}^{-}$ions on anatase (101) are lower than one $\mathrm{Cl}^{-}$ion $(0.99 \mathrm{eV})$ and two $\mathrm{Cl}^{-}$ions $(0.77 \mathrm{eV})$ on rutile (110), respectively; but at high $\mathrm{Cl}^{-}$concentration $\left(3 \mathrm{Cl}^{-}\right.$ions) on anatase (101) is higher $(0.45 \mathrm{eV})$ than that on rutile (110) $(0.43$ $\mathrm{eV})$. This indicates that the growth of anatase will be suppressed by the high concentration of $\mathrm{Cl}^{-}$, consistent with the present work result and the result of Hosono. ${ }^{56}$ Calculation results show that $\mathrm{SO}_{4}{ }^{2-}$ and $\mathrm{Cl}^{-}$selectively adsorb on anatase and rutile surface which suppress the rutile and anatase growth, respectively. The selectivity of adsorption is the key to control the phase ratio of the hierarchical structure.

To understand the structure-to-activity relationship, we synthesize pure anatase, rutile and anatase spheres by adjust $\mathrm{SO}_{4}{ }^{2-}, \mathrm{Cl}^{-}$and SDS. Irregular aggregated anatase octahedral crystals with no orientation (anatase powder, sample 1) were obtained when $0.1 \mathrm{M} \mathrm{H}_{2} \mathrm{SO}_{4}$ was added to the $0.15 \mathrm{M} \mathrm{TiCl}_{3}$ solution at $200{ }^{\circ} \mathrm{C}$ for $3 \mathrm{~h}$ (Fig. 7a). Rutile rod were obtained

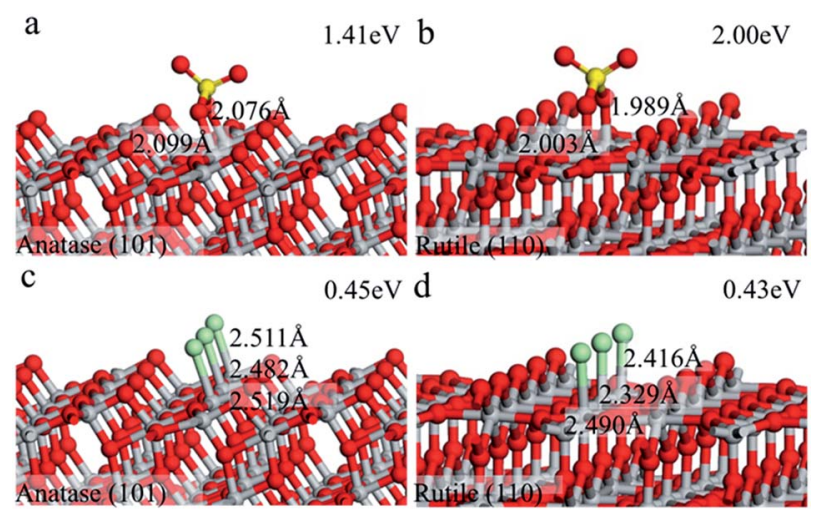

Fig. 6 Calculated structures (side view) of $\mathrm{SO}_{4}{ }^{2-}$ adsorption on anatase (101) surface (a) and rutile (110) surfaces (b); $\mathrm{Cl}^{-}$on (c) anatase (101) surface (c) and rutile (110) surfaces (d); the key bond lengths are signed and the absorption energy is labeled on the top surface. Ti: grey ball; O: red ball; S: yellow ball; Cl: green ball.
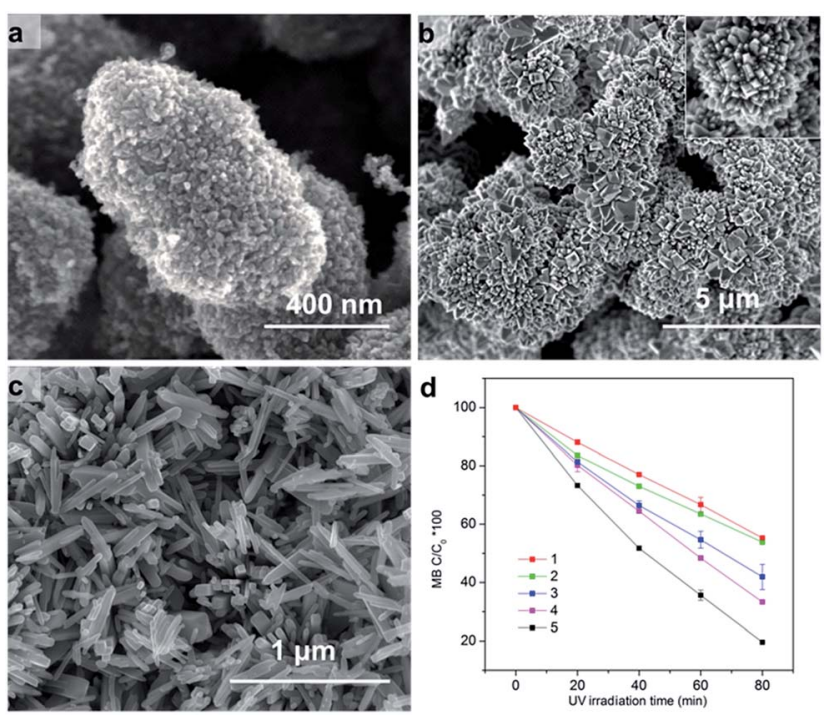

Fig. 7 FESEM images of $\mathrm{TiO}_{2}$ at $200{ }^{\circ} \mathrm{C}$ for $3 \mathrm{~h}$ with different additive. (a) $\mathrm{TiCl}_{3}$ concentration is $0.1 \mathrm{M}$, anatase powder $\left(0.1 \mathrm{M} \mathrm{H}_{2} \mathrm{SO}_{4}\right)$, (b) anatase sphere $\left(0.1 \mathrm{M} \mathrm{H}_{2} \mathrm{SO}_{4}, 0.012 \mathrm{M} \mathrm{SDS}\right)$, (c) rutile (5 M NaCl), (d) photocatalytic decomposition of MB under UV irradiation; (1) anatase powder, (2) rutile powder, (3) broken rutile/anatase sphere, (4) anatase sphere, (5) rutile/anatase bi-phase hierarchical structure.

(Fig. 7c) while $10 \mathrm{M} \mathrm{NaCl}$ was added to the $0.15 \mathrm{M} \mathrm{TiCl}_{3}$ solution (sample 2). The micro-sized anatase spheres assembled by nanometer size octahedral anatase crystals with orientation in [001] (sample 3) were obtained (Fig. 7b) when 0.012 M SDS was added to the $0.15 \mathrm{M} \mathrm{TiCl}_{3}$ solution at $200{ }^{\circ} \mathrm{C}$ for $3 \mathrm{~h}$. Sample 5 is rutile/anatase bi-phase hierarchical structure and sample 4 is the broken sample 5 by grind.

The activity performances photo-degradation $\mathrm{MB}$ of the five samples were evaluated. As Fig. 7d shown, the photoactivity of five samples. For the low electron-hole separation of the single phase, both anatase and rutile show lower photoactivity, and the photoactivity of anatase is very similar to that of rutile. However, the broken biphasic hierarchical architectures outperform the single counterpart one, and also the hierarchical bi-phase structure is superior to the single anatase sphere. The higher photoactivity performance is for the efficient charge separation of the phase junction. It also can be found that the photoactivity of hierarchical bi-phase architectures is higher than the broken counterpart, through the broken has higher specific surface area. The multi-reflections and charge separation is suppressed by the broken of the hierarchical structure and phase junction.

The photocatalytic activity order of the five tested samples is rutile/anatase hierarchical architectures > anatase sphere > broken rutile/anatase hierarchical architectures $>$ anatase powder $\approx$ rutile nanopins. The activity of rutile/anatase hierarchical architectures is about 2 times of the single phase powder. It was considered that the hollow structure possesses mesoporous networks, provides efficient transport pathways to their interior voids, endowing multi reflections of electromagnetic waves and high mass delivery speed which is critical for the greatly enhanced properties. And the performance of 
anatase (sphere-core)/rutile (nanopins-shell) with hollow hierarchical structure is superior to the single phase sphere. The high photocatalytic activity of the mixed-phase hierarchically structure should be attributed to structural and the phase junction.

\section{Conclusions}

In summary, a novel bi-phase hierarchical structure of anatase hollow sphere (core)/rutile nanopins (shell) was synthesized via a facile one-step hydrothermal synthesis strategy. The phase ratio is tunable by the adsorption agents. The successful synthesis of hierarchical structure via a controllable method, by introducing the SDS as soft template, opens up a new way to synthesize the hierarchical structure materials. The obtained biphase hierarchical structure contains anatase/rutile phase junction and hollow sphere, which possesses about 2 times higher activity of the single phase particles, have great potential for practical application.

\section{Conflicts of interest}

The authors declare no competing financial interest.

\section{Acknowledgements}

S.-C. Zhu is supported by NSFC (Grant No. 21703004); L. Fu is supported by Natural Science Foundation of Henan Department of Education (No. 16A330004), and the Natural Science Foundation of Nanyang Normal University (No. ZX2016003); Scientific research and service platform fund of Henan Province (2016151); the fund of scientific and technological innovation team of water ecological security for Water Source Region of Mid-line of South-to-North Diversion Project of Henan Province (17454).

\section{Notes and references}

1 D. Regonini, A. C. Teloeken, A. K. Alves, F. A. Berutti, K. Gajda-Schrantz, C. P. Bergmann, T. Graule and F. Clemens, ACS Appl. Mater. Interfaces, 2013, 5, 1174711755.

2 P. Hartmann, D.-K. Lee, B. M. Smarsly and J. Janek, ACS Nano, 2010, 4, 3147-3154.

3 C. Gomes Silva, R. Juarez, T. Marino, R. Molinari and H. Garcia, J. Am. Chem. Soc., 2011, 133, 595-602.

4 S. Neatu, J. Antonio Macia-Agullo, P. Concepcion and H. Garcia, J. Am. Chem. Soc., 2014, 136, 15969-15976.

5 C. L. Anfuso, R. C. Snoeberger III, A. M. Ricks, W. Liu, D. Xiao, V. S. Batista and T. Lian, J. Am. Chem. Soc., 2011, 133, 6922-6925.

6 R. Chalasani and S. Vasudevan, ACS Nano, 2013, 7, 40934104.

7 W. J. Youngblood, S. H. A. Lee, K. Maeda and T. E. Mallouk, Acc. Chem. Res., 2009, 42, 1966-1973.

8 X. B. Chen, S. H. Shen, L. J. Guo and S. S. Mao, Chem. Rev., 2010, 110, 6503-6570.
9 A. I. Hochbaum and P. Yang, Chem. Rev., 2009, 110, 527-546.

10 K. Maeda, K. Teramura, D. Lu, T. Takata, N. Saito, Y. Inoue and K. Domen, Nature, 2006, 440, 295.

11 J. Zhu, S.-H. Wang, Z.-F. Bian, C.-L. Cai and H.-X. Li, Res. Chem. Intermed., 2009, 35, 769-777.

12 T.-D. Nguyen-Phan, E. J. Kim, S. H. Hahn, W.-J. Kim and E. W. Shin, J. Colloid Interface Sci., 2011, 356, 138-144.

13 M. Feng, W. You, Z. S. Wu, Q. D. Chen and H. B. Zhan, ACS Appl. Mater. Interfaces, 2013, 5, 12654-12662.

14 W. Li, Y. Deng, Z. Wu, X. Qian, J. Yang, Y. Wang, D. Gu, F. Zhang, B. Tu and D. Zhao, J. Am. Chem. Soc., 2011, 133, 15830-15833.

15 J. Zhang, S. R. Wang, M. J. Xu, Y. Wang, B. L. Zhu, S. M. Zhang, W. P. Huang and S. H. Wu, Cryst. Growth Des., 2009, 9, 3532-3537.

16 X. F. Qu, Q. Z. Yao, G. T. Zhou, S. Q. Fu and J. L. Huang, J. Phys. Chem. C, 2010, 114, 8734-8740.

17 L. Zhang, H. B. Wu, S. Madhavi, H. H. Hng and X. W. Lou, J. Am. Chem. Soc., 2012, 134, 17388-17391.

18 G. X. Zhu, H. Xu, Y. Y. Xiao, Y. J. Liu, A. H. Yuan and X. P. Shen, ACS Appl. Mater. Interfaces, 2012, 4, 744-751.

19 Z. Q. Sun, J. H. Kim, Y. Zhao, F. Bijarbooneh, V. Malgras, Y. Lee, Y. M. Kang and S. X. Dou, J. Am. Chem. Soc., 2011, 133, 19314-19317.

20 M. D. Ye, H. Y. Liu, C. J. Lin and Z. Q. Lin, Small, 2013, 9, 312-321.

21 Y. Liu, Y. H. Deng, Z. K. Sun, J. Wei, G. F. Zheng, A. M. Asiri, S. B. Khan, M. M. Rahman and D. Y. Zhao, Small, 2013, 9, 2702-2708.

22 Q. Li, E. T. Liu, Z. Lu, H. Yang and R. Chen, Mater. Lett., 2014, 130, 115-119.

23 H. Shi, X. Xiao, L. Zeng, Q. Zhang, J. Nan and L. Wang, J. Nanosci. Nanotechnol., 2014, 14, 6934-6940.

24 H. X. Li, Z. F. Bian, J. Zhu, D. Q. Zhang, G. S. Li, Y. N. Huo, H. Li and Y. F. Lu, J. Am. Chem. Soc., 2007, 129, 8406-8407.

25 M. Ye, H.-Y. Liu, C. Lin and Z. Lin, Small, 2013, 9, 312-321.

26 L. Zhang, D. Jing, X. She, H. Liu, D. Yang, Y. Lu, J. Li, Z. Zheng and L. Guo, J. Mater. Chem. A, 2014, 2, 2071-2078.

27 Y. Zhu, J. Ren, X. Yang, G. Chang, Y. Bu, G. Wei, W. Han and D. Yang, J. Mater. Chem. A, 2017, 5, 9952-9959.

28 T. Kawahara, Y. Konishi, H. Tada, N. Tohge, J. Nishii and S. Ito, Angew. Chem., 2002, 114, 2935-2937.

29 J. Zhang, Q. Xu, Z. Feng, M. Li and C. Li, Angew. Chem., Int. Ed., 2008, 47, 1766-1769.

30 L. Cao, D. H. Chen, W. Li and R. A. Caruso, ACS Appl. Mater. Interfaces, 2014, 6, 13129-13137.

31 R. Su, R. Bechstein, L. So, R. T. Vang, M. Sillassen, B. Esbjornsson, A. Palmqvist and F. Besenbacher, J. Phys. Chem. C, 2011, 115, 24287-24292.

32 S. C. Zhu, S. H. Xie and Z. P. Liu, J. Phys. Chem. Lett., 2014, 5, 3162-3168.

33 B. Liu, A. Khare and E. S. Aydil, ACS Appl. Mater. Interfaces, 2011, 3, 4444-4450.

34 D. Yang, H. Liu, Z. Zheng, Y. Yuan, J.-C. Zhao, E. R. Waclawik, X. Ke and H. Zhu, J. Am. Chem. Soc., 2009, 131, 17885-17893. 
35 H.-L. Kuo, C.-Y. Kuo, C.-H. Liu, J.-H. Chao and C.-H. Lin, Catal. Lett., 2007, 113, 7-12.

36 H. U. Lee, Y. C. Lee, S. C. Lee, S. Y. Park, B. Son, J. W. Lee, C. H. Lim, C. J. Choi, M. H. Choi, S. Y. Lee, Y. K. Oh and J. Lee, Chem. Eng. J., 2014, 254, 268-275.

37 W. N. Zhao, S. C. Zhu, Y. F. Li and Z. P. Liu, Chem. Sci., 2015, 6, 3483-3494.

38 R. Ostermann, D. Li, Y. D. Yin, J. T. McCann and Y. N. Xia, Nano Lett., 2006, 6, 1297-1302.

39 Y. Li, N. Koshizaki, H. Q. Wang and Y. Shimizu, ACS Nano, 2011, 5, 9403-9412.

40 J. M. Wang, P. S. Lee and J. Ma, Cryst. Growth Des., 2009, 9, 2293-2299.

41 J. Zhang, Y. Tang, K. Lee and O. Min, Science, 2010, 327, 1634-1638.

42 E. Hosono, S. Fujihara, H. Lmai, I. Honma, I. Masaki and H. S. Zhou, ACS Nano, 2007, 1, 273-278.

43 J. M. Soler, E. Artacho, J. D. Gale, A. Garcia, J. Junquera, P. Ordejon and D. Sanchez-Portal, J. Phys.: Condens. Matter, 2002, 14, 2745-2779.

44 J. Junquera, O. Paz, D. Sanchez-Portal and E. Artacho, Phys. Rev. B, 2001, 64, 235111-235119.
45 N. Troullier and J. L. Martins, Phys. Rev. B: Condens. Matter Mater. Phys., 1991, 43, 1993-2006.

46 J. P. Perdew, K. Burke and M. Ernzerhof, Phys. Rev. Lett., 1996, 77, 3865-3868.

47 J. L. Fattebert and F. Gygi, Phys. Rev. B: Condens. Matter Mater. Phys., 2006, 73, 115124.

48 H.-F. Wang and Z.-P. Liu, J. Phys. Chem. C, 2009, 113, 1750217508.

49 Y.-H. Fang and Z.-P. Liu, J. Am. Chem. Soc., 2010, 132, 1821418222.

50 Y.-H. Fang, G.-F. Wei and Z.-P. Liu, Catal. Today, 2013, 202, 98-104.

51 W. N. Zhao and Z. P. Liu, Chem. Sci., 2014, 5, 2256-2264.

52 C. Shang, X. J. Zhang and Z. P. Liu, Phys. Chem. Chem. Phys., 2014, 16, 17845-17856.

53 S.-C. Zhu, S.-H. Guan, W.-N. Zhao and Z.-P. Liu, Top. Catal., 2015, 644.

54 C. J. Niu, J. S. Meng, C. H. Han, K. N. Zhao, M. Y. Yan and L. Q. Mai, Nano Lett., 2014, 14, 2873-2878.

55 X. Bokhimi, A. Morales, E. Ortiz, T. López, R. Gómez and J. Navarrete, J. Sol-Gel Sci. Technol., 2004, 29, 31-40.

56 E. Hosono, S. Fujihara, K. Kakiuchi and H. Imai, J. Am. Chem. Soc., 2004, 126, 7790-7791. 